\title{
'Super-Innervation' of a Free Latissimus Dorsi Flap in Facial Reanimation: A Novel Strategy for Supplementing Muscle Innervation
}

\author{
Leckenby J*, Branford OA, Ghali S and Grobbelaar AO \\ Department of Plastic Surgery, Royal Free Hampstead NHS Trust, Pond Street, London, NW3 2QG, UK
}

${ }^{*}$ Corresponding author: Leckenby J, Department of Plastic Surgery, Royal Free Hampstead NHS Trust, Pond Street, London, NW3 2QG, United Kingdom, Tel: + 44207794 0500, Fax: +44 207 8302468, E-mail: joleckenby@ gmail.com

Citation: Leckenby J, Branford OA, Ghali S, Grobbelaar AO (2014) ‘Super-Innervation’ of a Free Latissimus Dorsi Flap in Facial Reanimation: A Novel Strategy for Supplementing Muscle Innervation. SAJ Cas Rep 1: 202. doi: $10.18875 / 2375-7043.1 .202$

Article history: Received: 07 August 2014, Accepted: 04 November 2014, Published: 06 November 2014

\begin{abstract}
The gold-standard for facial reanimation in facial palsy involves a first stage crossed facial nerve graft and a second stage free muscle flap transfer, with a single neurorrhaphy of the muscle flap nerve to the nerve graft. The timing of the second stage is usually 6 months after the first stage, which is undertaken once there is a positive Tinnel's sign. We present new technique for situations where there is either a significant delay between the first and second stages or if there is a question over the viability of a nerve graft. To maximise the chances of success a latissimus dorsi free flap was used, where the medial branch of the thoracodorsal nerve was joined to the cross facial nerve graft and the lateral branch was 'super-innervated' by the nerve to masseter. Although both these techniques have been described, to our knowledge they have not been used previously in the same patient.
\end{abstract}

Keywords: CHARGE Syndrome; Facial Palsy; Free Flap; Nerve Grafting; Super-Innervation; SuperCharging

\section{Introduction}

Facial reanimation can be performed in a single stage or two-staged procedure using free functional muscle transfer [1]. In a one stage procedure the muscle is harvested and transported to the affected side of the face. At this stage a single neurorrhaphy is performed between the nerve from the muscle pedicle to a branch of a functioning cranial nerve, usually the masseteric branch of the trigeminal nerve or to a branch of the hypoglossal nerve [2]; this results in a voluntary smile upon biting (masseteric branch) or swallowing (hypoglossal nerve) and is reliant on brain plasticity. Alternatively, a one stage procedure can be performed by harvesting a muscle with a long neurovascular pedicle and performing a neurorrhaphy to a very distal branch of the contra-lateral functioning facial nerve; commonly the bulkier muscles of latissimus dorsi and gracilis are used with the possibility of creating a more noticeable incision around the contralateral naso-labial fold or parotid area [3].

A two stage reanimation procedure differs by utilizing the functioning contra-lateral facial nerve which ultimately results in the spontaneous ability to smile [1]. The first stage is usually planned between four and eight years of age and involves harvesting a nerve graft, usually the sural nerve, performing a neurorrhaphy to a buccal branch of the functioning contra-lateral facial nerve and tunnelling the graft sub-cutaneously to the pre-auricular region of the non-functioning side. Typically, axonal regeneration does not take place for approximately six weeks within the cross-facial nerve graft (CFNG) and then continues at approximately $1 \mathrm{~mm}$ per day. Nerve growth can be monitored clinically with Tinel's sign, however in up to $25 \%$ this may be negative despite actual axonal growth as this is a more reliable test of sensory nerve regeneration [4], and if a diagnostic guarantee is required a biopsy of the CFNG may be taken and histo-morphological assessment may be performed although this adds an additional operation in the patient's management. Once satisfactory evidence has been established the second stage can be planned. The second stage involves harvesting a muscle with its neurovascular pedicle and transporting it to the non-functioning side. Anastomoses are performed to the facial vessels and neurorrhaphy is performed to the CFNG. Post-operatively re-innervation of the free muscle transfer can begin to be seen between three and six months. 
The three most common choices of muscle for the second stage free transfer are pectoralis minor, gracilis and latissimus dorsi. Each muscle has its advantages and disadvantages which are based around bulkiness of the flap, pedicle length and donor site morbidity. The preferred choice in our unit is to harvest pectoralis minor [1,5]. This provides a good size match, excellent vector and minimal functional deficit at the expense of a shorter pedicle length. Both the latissimus dorsi and gracilis offer longer neurovascular pedicles but may lead to bulkier flaps, however the latissimus dorsi offers the additional benefit of being segmentally innervated allowing sections of the muscle to be used in isolation [6].

There are many challenges which can cause problems for a facial palsy surgeon. Previous facial surgery can result in scarring and the exact understanding of what anatomy is available is troublesome. In the paediatric population, co-existing developmental anomalies can also lead to unpredictable anatomy. In congenital patients, facial palsy is normally only one part of an extensive syndrome and co-morbidities and the timing of other surgery plays a role in planning a facial reanimation procedure.

\section{Surgical Technique}

A patient presented to our unit with a right-sided facial palsy as a result of a congenital Charge syndrome. Charge association was first described in 1981 by Pagon [7] and Blake [8] proposed that at least either four major features or three major and three minor features must be present, these are summarised in Table 1.

\begin{tabular}{|c|c|}
\hline Major Criteria & Minor Criteria \\
\hline Coloboma & Characteristic facial dysmorphology \\
\hline Choanal atresia & Facial clefting \\
\hline Cranial nerve abnormalities & Traceoesophageal fistula \\
\hline Auditory anomalies & Congenital heart defects \\
\hline & Genitourinary anomalies \\
\hline & Developmental delay \\
\hline
\end{tabular}

Table 1: Clinical features of Charge Syndrome

The patient underwent the first stage of facial reanimation using a sural cross-facial nerve graft at age 11 years. Due to recurrent chest infections the second stage was repeatedly delayed until 3 years after the first stage. The Tinel's sign, which had become positive, had been lost. Although no time limit has been proposed for the maximum delay after which the crossed facial nerve graft (CFNG) becomes unusable, the lack of neurotrophic stimuli resulting from an open-ended nerve graft is likely to cause axonal degeneration [9]. This will increase with time and a four-year period is generally deemed to be the maximum. To increase the chances of success a latissimus dorsi free flap was used as the thoracodorsal nerve can be separated into medial and lateral branches: the medial branch was coapted to the CFNG and the lateral branch was 'super-innervated' by the nerve to the masseter muscle (Figure 1 and 2). Masseteric nerve innervation has recently become popular as a primary nerve source for unilateral facial palsy [10] however, questions regarding brain plasticity remain unresolved. The utilisation of a CFNG to supply axonal reinnervation of a FFMT in a two-stage procedure is well established. Similarly, the use of the masseteric nerve as a donor for FFMT in one-stage procedures is commonplace. To our knowledge these two techniques have not been combined at the same operation to supply a FFMT. This is a novel strategy to protect the transferred muscle against end-plate degeneration and to provide a spontaneous smile.

\section{Discussion}

In this case the latissimus dorsi was chosen for the greater pedicle length and due to the thoracodorsal nerve dividing into two branches to supply the muscle [11]. Due to the delay in timing of surgery as a consequence of the patient's comorbidities and also the fact there was no positive Tinel's sign, it was uncertain as to whether the CFNG was functioning. A plan was made to perform an additional neurorrhaphy to the masseteric branch of the trigeminal nerve $(\mathrm{CN} \mathrm{V})$; this would require a longer pedicle than pectoralis minor can offer, and the muscle has only one nerve branch supplying it.

The blood supply of the latissimus dorsi muscle is via the thoracodorsal artery, a branch of the circumflex scapular artery, and its nerve supply is via the thoracodorsal nerve. The nerve divides proximal to the artery and the branches have been shown to diverge separately [12]. During the surgery the lastissimus dorsi was raised through an axillary incision. The thoracodorsal nerve was identified, running with the thoracodorsal artery and the medial and lateral branches of the nerve were identified with the lateral branch being divided from the main nerve. Both the lateral and medial branches were independently stimulated intraoperatively and contraction of the entire muscle bulk by each branch was demonstrated. Two end-to-end neurorrhaphies were performed, one between the medial branch and the CFNG and the lateral branch to the masseteric branch of the trigeminal nerve. This resulted in a spontaneous smile via the CFNG (CN VII) with re-inforcement and voluntary contraction via the masseteric branch (CN V) (Figure 3). Initially distinguishing the contribution of individual nerves supplying the FFMT was difficult and it appeared that the masseteric innervation of the FFMT began to appear at 3-months and the facial nerve via the CFNG at 8-months. Two-years postoperatively the patient could produce a bilateral smile or a unilateral smile of the FFMT. 


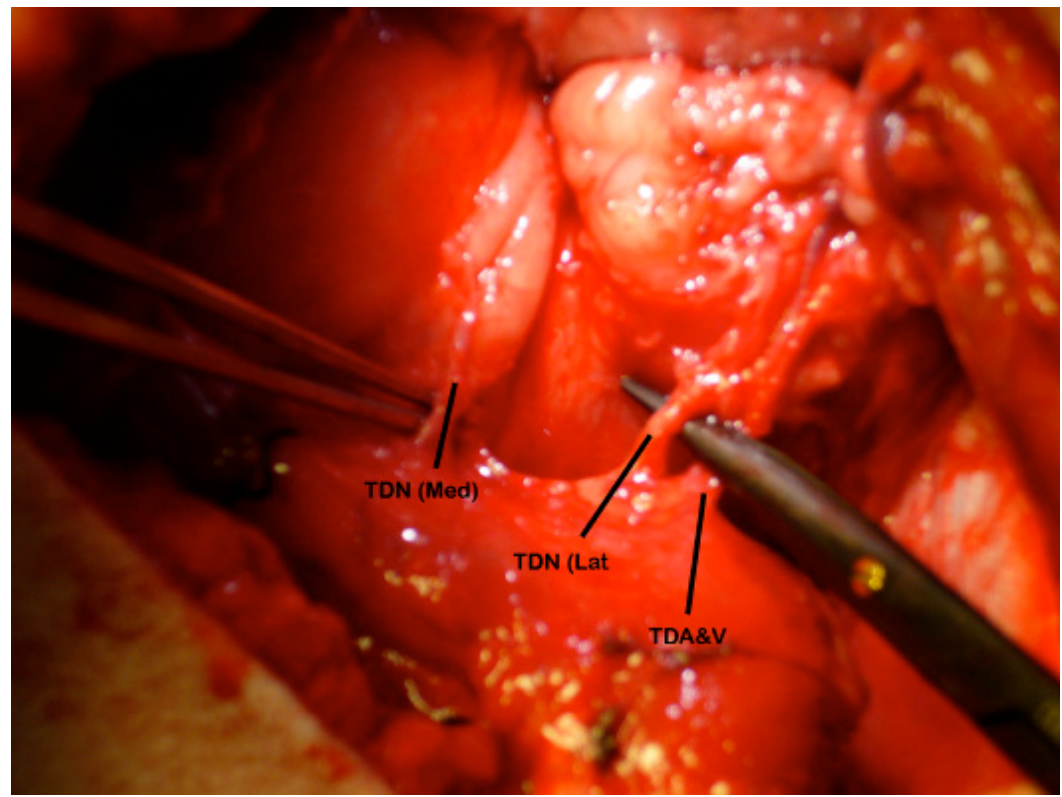

Figure 1: Anatomy of the latissimus dorsi neurovascular pedicle. (A) An intraoperative photograph of the arrangement of the neurovascular pedicle showing the organisation of the two branches of the thoracodorsal nerve (TDN); The medial branch (TDN(Med)) and the lateral branch (TDN(Lat)) can be seen in relation to the thoracodorsal artery and vein (TDA\&V).
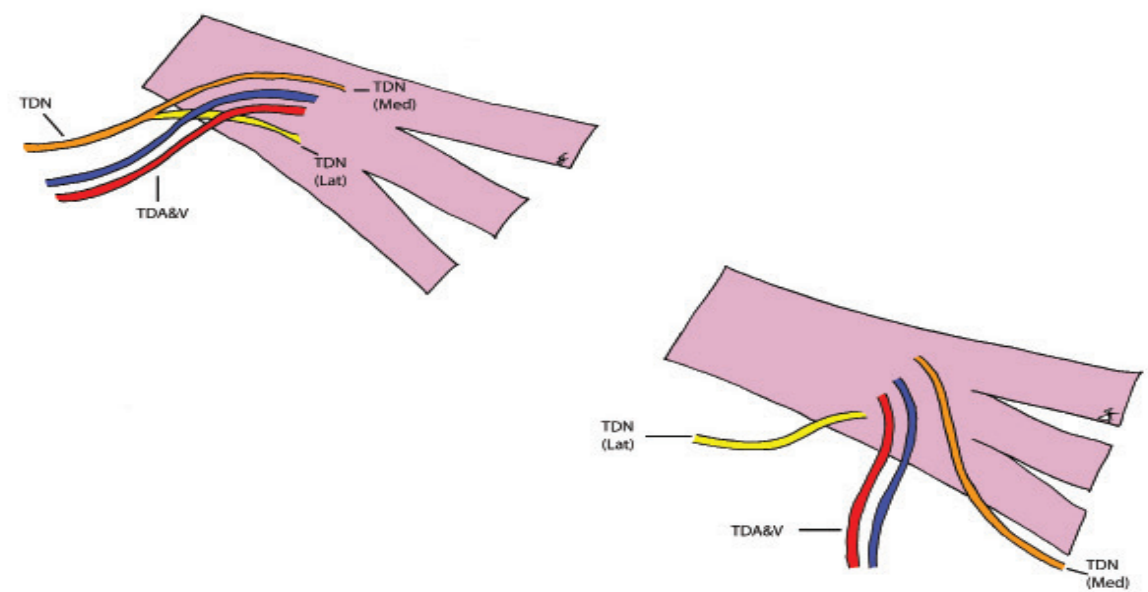

Figure 1 (B): A diagrammatic representation of the neurovascular pedicle before and after the TDN (Lat) has been separated from the main trunk.

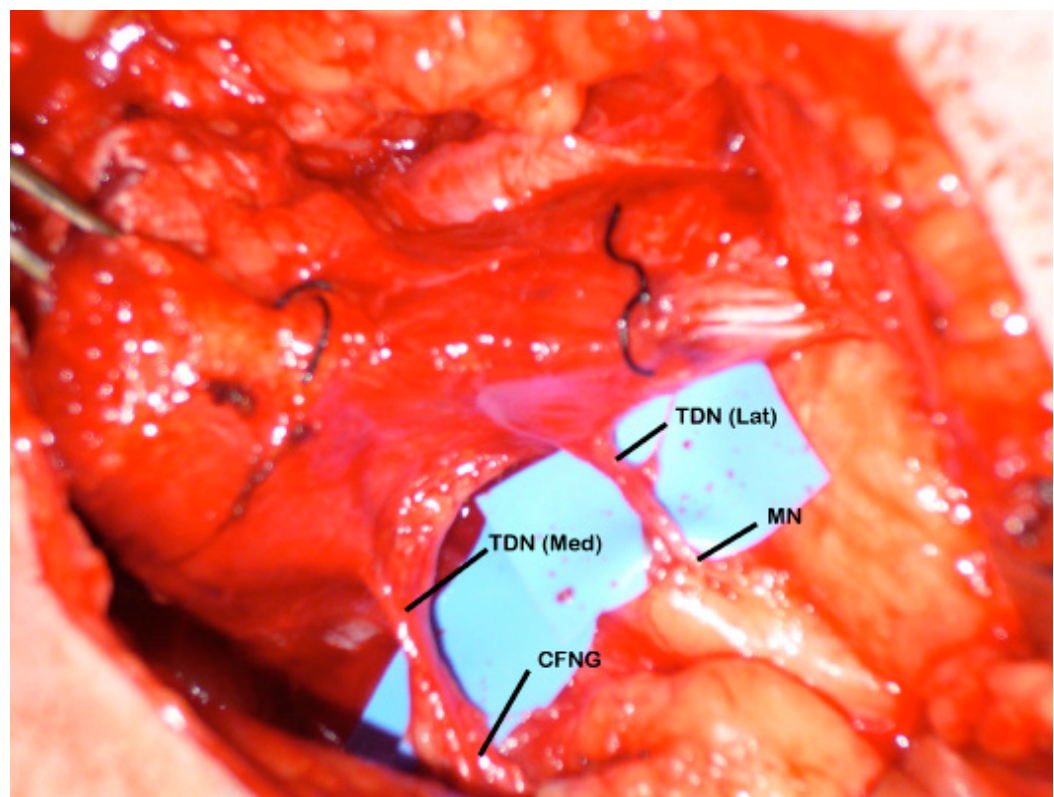

Figure 2: Insetting of the latissimus dorsi free functional muscle flap. (A) An intraoperative photograph depicting the insetting of the latissimus dorsi muscle and the joining of the two branches of the thoracodorsal nerve (TDN). The TDN (Med) was coapted to the cross facial nerve graft (CFNG) and the TDN (Lat) was joined to the nerve to the masseter muscle (MN) and thus 'super-innervating' the muscle flap. 


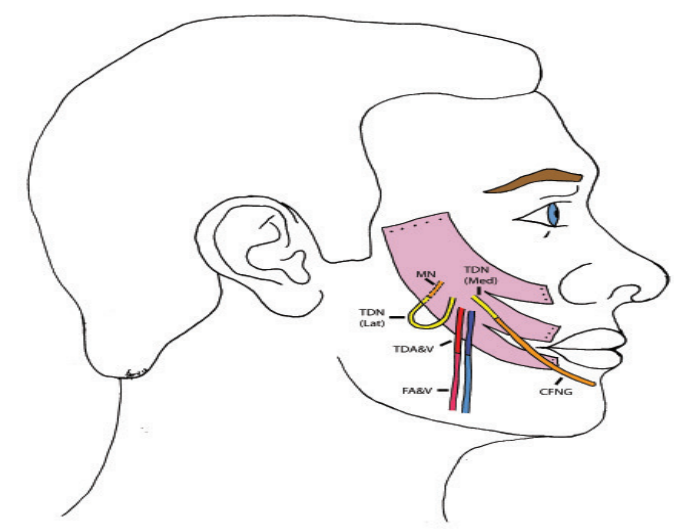

Figure 2(B): A diagrammatic illustration showing the inset of the flap, in addition to the above, showing the thoracodorsal artery and vein $(T D A \& V)$ being anastomosed to the facial artery and vein $(F A \& V)$. Note that the CFNG passes beneath the lower lip due to the patient having previous cleft surgery.

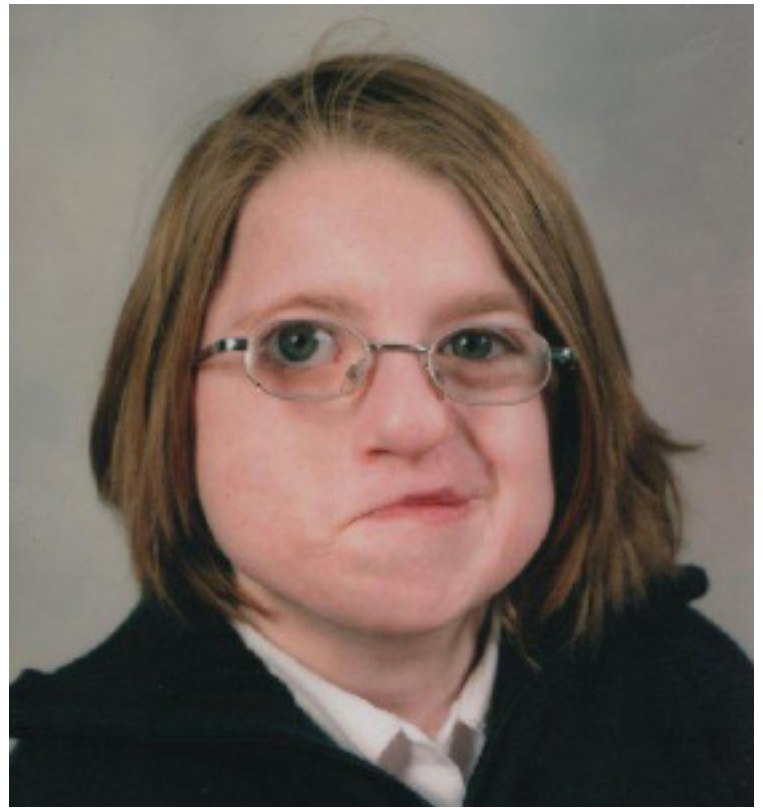

Figure 3: Pre and postoperative photographs. (A) Pre operative photography of a patient with CHARGE syndrome and complete right sided facial paralysis.

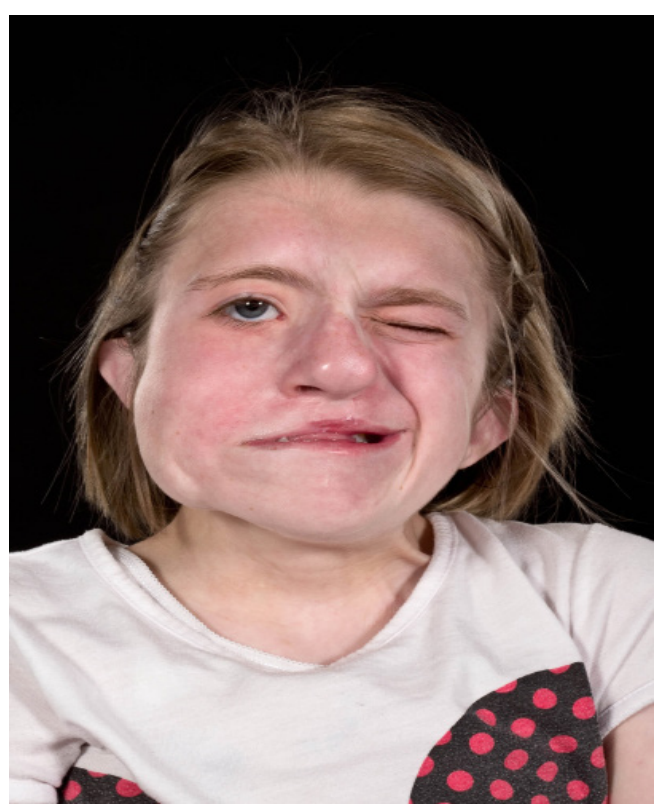

Figure 3(B): A 1 yr post operative photograph showing facial reanimation with good excursion of the muscle when both smiling and biting. 
The concept of 'supercharging' a free flap is well described [13]. This involves the anastomosis of two arteries in order to improve blood supply to a flap. To our knowledge, the concept of performing two end-to-end neurorrhaphies as a 'super-innervation' of a single free muscle flap has not yet been described. This novel technique may be useful in cases where the chance of the muscle flap reinnervation needs to be maximized, in this case indicated by the loss of the positive Tinel's sign. Watanabe and colleagues have described a dual innervation of a free latissimus dorsi muscle transfer for facial reanimation [14]. However they did not perform a second neurorrhaphy and the reinnervation relied on neurotization through the proximity to the masseteric muscle, which may be unreliable. Yamamoto and colleagues introduced the concept of supercharging the nerve supply in facial palsy using an end-toside technique [15], however, end-to-end anastomosis provides maximal axonal regeneration [16]. We propose that in the present case the CFNG is being supplemented by the masseteric nerve resulting in the ability to smile spontaneously being reinforced by voluntary muscle contraction via the trigeminal branch. This serves as a useful new surgical application of the latissimus dorsi muscle and may play a role in reducing end-plate degeneration of the muscle whilst being reinnervated by the CFNG.

\section{References}

1. Ghali S, MacQuillan A, Grobbelaar AO (2011) Reanimation of the middle and lower face in facial paralysis: review of the literature and personal approach. J Plast Reconstr Aesthet Surg 64: 423-31.

2. Terzis JK, Konofaos P (2008) Nerve transfers in facial palsy. Facial Plast Surg 24: 177-93.

3. Harii K, Asato H, Yoshimura K, Sugawara Y, Nakatsuka T et al. (1998) One-stage transfer of the latissimus dorsi muscle for reanimation of a paralyzed face: a new alternative. Plast Reconstr Surg 102: 941-51.

4. Harrison DH (1985) Pectoralis minor for facial palsy. Plast Reconstr Surg. 76: 656-7.

5. Terzis JK (1989) Pectoralis minor: a unique muscle for correction of facial palsy. Plast Reconstr Surg 83: 767-76.

6. Mackinnon SE, Dellon AL (1988) Technical considerations of the latissimus dorsi muscle flap: a segmentally innervated muscle transfer for facial reanimation. Microsurgery 9: 36-45.

7. Pagon RA, Graham JM, Zonana J, Yong SL (1981) Coloboma, congenital heart disease, and choanal atresia with multiple anomalies: CHARGE association. J Pediatr 99: 223-7.

8. Blake KD, Davenport SL, Hall BD, Hefner MA, Pagon RA et al. (1998) CHARGE association: an update and review for the primary pediatrician. Clin Pediatr (Phila) 37: 159-73.

9. Bevan S, JH. Steinbach (1983) Denervation increases the degradation rate of acetylcholine receptors at end-plates in vivo and in vitro. J Physiol 336: 159-77.

10. Bae YC, Zuker RM, Manktelow RT, Wade S (2006) A comparison of commissure excursion following gracilis muscle transplantation for facial paralysis using a cross-face nerve graft versus the motor nerve to the masseter nerve. Plast Reconstr Surg 117: 2407-13.

11. Dellon A.L, S.E. Mackinnon (1985) Segmentally innervated latissimus dorsi muscle. Microsurgical transfer for facial reanimation. J Reconstr Microsurg 2: 7-12.

12. Ferguson LD, Paterson T, Ramsay F, Arrol K, Dabernig J et al. (2011) Applied anatomy of the latissimus dorsi free flap for refinement in one-stage facial reanimation. J Plast Reconstr Aesthet Surg 64: 1417-23.

13. Beegle PH (1990) Microvascular augmentation of TRAM flap circulation ("supercharged” TRAM), ed. C.R. Hartrampf: Raven Press.

14. Watanabe Y, Akizuki T, Ozawa T, Yoshimura K, Agawa K, et al. (2009) Dual innervation method using one-stage reconstruction with free latissimus dorsi muscle transfer for re-animation of established facial paralysis: simultaneous reinnervation of the ipsilateral masseter motor nerve and the contralateral facial nerve to improve the quality of smile and emotional facial expressions. J Plast Reconstr Aesthet Surg 62: 1589-97.

15. Furukawa H, Saito A, Mol W, Sekido M, Sasaki S, et al. (2008) Double innervation occurs in the facial mimetic muscles after facial-hypoglossal end-to-side neural repair: rat model for neural supercharge concept. J Plast Reconstr Aesthet Surg 61: 257-64.

16. Jaeger MR, Braga-Silva J, Gehlen D, Pereira-Filho Gde A, Zettler CG, et al. (2011) End-to-end versus end-to-side motor and sensory neurorrhaphy in the repair of the acute muscle denervation. Ann Plast Surg 67: 391-6. 